\title{
A multicentre prospective randomized equivalence trial of a soft bandage and immediate discharge versus current treatment with rigid immobilization for torus fractures of the distal radius in children
}

PROTOCOL FOR THE FOREARM FRACTURE RECOVERY IN CHILDREN EVALUATION (FORCE) TRIAL

\section{J. Achten, \\ R. Knight, \\ S. J. Dutton, \\ M. L. Costa, \\ J. Mason, \\ M. Dritsaki, \\ D. Appelbe, \\ S. Messahel, \\ D. Roland, \\ J. Widnall, \\ D. C. Perry}

University of Oxford, Oxford, UK

Correspondence should be sent to Daniel C Perry; email:

daniel.perry@ndorms.ox.ac.uk

doi: 10.1302/2046-3758.16.BJO2020-0014.R1

Bone Joint Open 2020;1-6:214221.

\begin{abstract}
Aims
Torus fractures are the most common childhood fracture, accounting for 500,000 UK emergency attendances per year. UK treatment varies widely due to lack of scientific evidence. This is the protocol for a randomized controlled equivalence trial of 'the offer of a soft bandage and immediate discharge' versus 'rigid immobilization and follow-up as per the protocol of the treating centre' in the treatment of torus fractures .

\section{Methods}

Children aged four to 15-years-old inclusive who have sustained a torus/buckle fracture of the distal radius with/without an injury to the ulna are eligible to take part. Baseline pain as measured by the Wong Baker FACES pain scale, function using the Patient-Reported Outcomes Measurement Information System (PROMIS) upper limb, and quality of life (QoL) assessed with the EuroQol EQ-5D-Y will be collected. Each patient will be randomly allocated (1:1, stratified by centre and age group (four to seven years and $\geq$ eight years) to either a regimen of the offer of a soft bandage and immediate discharge or rigid immobilization and follow-up as per the protocol of the treating centre.
\end{abstract}

\section{Results}

At day one, three, and seven, data on pain, function, QoL, immobilization, and analgesia will be collected. Three and six weeks after injury, the main outcomes plus data on complications, resource use, and school absence will be collected. The primary outcome is the WongBaker FACES pain scale at three days post-randomization. All data will be obtained through electronic questionnaires completed by the participants and/or parents/guardian.

Cite this article: Bone Joint Open 2020;1-6:214-221.

Keywords: trauma, orthopaedics, paediatrics, fracture, randomised clinical trial

Strength and limitations

- Broad eligibility criteria to ensure generalizability.

- Patient-centred outcome data.

- Assessment of outcomes at multiple time points will allow for information on recovery profile.
- In addition to a comparison of clinical outcomes, a health economic evaluation will be performed.

- It will not be possible to blind patients and care givers to their allocated treatment. 


\section{Introduction}

Torus (buckle) fractures of the distal radius are the most common fractures in children, with the bone 'buckling', so there is deformation without a break in the cortex. They result from trauma to growing bones and account for 500,000 UK emergency attendances annually. ${ }^{1}$

They are very low risk injuries for complications or deformity in the skeletally immature, and these fractures universally heal well. ${ }^{2}$

There is considerable variation in the management of torus fractures. Treatment varies from the use of a removable rigid splint, to plaster cast immobilization, to more flexible splints. The variation in practice has arisen from a longstanding taught doctrine of rigid immobilization for fractures, ${ }^{3}$ tempered with newer evidence to suggest that simpler treatment methods are frequently as effective or perhaps even more effective. ${ }^{4-8}$ The proponents of rigid forms of immobilization (i.e. cast/splint) argue that this maximizes pain relief, and minimises the occurrence of complications, i.e. refracture. However, there is growing evidence to support the absence of complications with growing acceptance that rigid immobilization may not improve pain control but will unduly restrict function, and that patients may safely be discharged at diagnosis. ${ }^{7,9}$ Two systematic reviews support the abandonment of non-removable rigid casts in favour of splints removable at home. ${ }^{10,11}$

The recent NICE non-complex fracture guidelines made recommendations on the management of these injuries. ${ }^{1}$ The interventions considered as part of this review were non-removable rigid casts (i.e. fibreglass/plaster of Paris), soft casts, removable splints and bandaging. The NICE review group concluded that bandaging was probably the optimal treatment approach, due to the convenience, adequate pain control and the ability to promote early function. The NICE review concluded that torus fractures of the distal radius should not be immobilized in a non-removable rigid cast, and advocated discharge from the emergency department without a subsequent need for outpatient follow-up. NICE questioned whether any intervention was necessary at all, and subsequently recommended a trial to determine the optimal intervention. To summarize, there are several options for the treatment of torus fractures of the distal radius, with key differences relating to the degree of immobilization provided, and the follow-up required. Soft bandaging restricts movement the least and may promote early function, but concern remains about pain and the potential for complications, despite evidence to the contrary. This is the protocol of a randomized equivalence trial of soft bandage with immediate discharge versus immobilization with a splint as per current practice.

The trial will be reported in line with the CONSORT statement using the non-pharmacological treatment interventions and patient reported outcomes extensions. ${ }^{12,13}$

\section{Aims}

The aim of this project is to establish whether treating children with a torus fracture of the distal radius with the offer of a soft bandage and immediate discharge provides the same recovery, in terms of pain and function, as treating them with rigid splint immobilization and follow-up as per the protocol of the treating centre.

The primary objective is:

To quantify and draw inferences on observed differences in the Wong-Baker FACES Pain Rating Scale (Wong-Baker scale) between soft bandage and immediate discharge versus rigid immobilization and standard follow-up, at three days post-randomization.

The secondary objectives are:

1. To assess differences in the Wong-Baker scale between trial treatment groups at one day, seven days, three weeks, and six weeks post randomization

2. To determine differences in the use of regular analgesia between trial treatment groups at one day, three days, and seven days post-randomization.

3. To quantify and draw inferences on functional recovery using the PROMIS Upper Limb Limb Score for Children Computer Adaptive Test (a validated measure of childhood upper limb function) between the trial treatment groups at three days, seven days, three weeks, and six-weeks post-randomization

4. To quantify and draw inferences on observed differences in the EQ-5DY (a validated assessment of healthrelated quality of life) between trial treatment groups at three days, seven days, three weeks, and six-weeks post-randomization.

5. To determine differences in the number of days of school absence between trial treatment groups up to six-weeks post-randomization.

6. To determine differences in the complication rate between trial treatment groups, including the need for further hospital attendance up to six-weeks post-randomization.

7. To investigate, using appropriate statistical and economic analysis methods, the resource use, and comparative cost-effectiveness between trial treatment groups during the first six weeks after injury.

Outcome measures. A schedule of data collection is outlined in Table I.

To determine the most appropriate primary outcome we discussed the proposed trial with children and families affected by torus fractures of the distal radii, and consulted fifteen members of the GenerationR NIHR young person's advisory group (YPAG). Children indicated that pain was the most important outcome, particularly in the first few days after the injury, with early function as a secondary concern. Parents were similarly concerned by pain, function and in addition the duration of school absence. With input from these 
Table I. Outcomes collection schedule.

\begin{tabular}{|c|c|c|}
\hline Baseline & $\begin{array}{l}\text { Wong-Baker FACES } \\
\text { Pain Scale, } \\
\text { PROMIS upper limb } \\
\text { (UE) for Children } \\
\text { EQ-5D-Y }\end{array}$ & $\begin{array}{l}\text { Prior to } \\
\text { randomization in } \\
\text { the emergency } \\
\text { department. }\end{array}$ \\
\hline Day 1 & $\begin{array}{l}\text { Wong-Baker FACES Pain } \\
\text { Scale, } \\
\text { Use of immobilization } \\
(\mathrm{Y} / \mathrm{N}) \text {, } \\
\text { Analgesia } \mathrm{Y} / \mathrm{N} \\
\text { Treatment satisfaction }\end{array}$ & Electronic collection \\
\hline Day 3 & $\begin{array}{l}\text { Wong-Baker FACES } \\
\text { Pain scale (primary } \\
\text { outcome), } \\
\text { PROMIS-UE, } \\
\text { EQ-5D-Y, } \\
\text { Use of immobilization } \\
(\mathrm{Y} / \mathrm{N}) \text {, } \\
\text { Use of Analgesia }(\mathrm{Y} / \mathrm{N}) \text {. }\end{array}$ & Electronic collection \\
\hline Day 7 & $\begin{array}{l}\text { Wong-Baker FACES Pain } \\
\text { scale, } \\
\text { PROMIS-UE, } \\
\text { EQ-5D-Y, } \\
\text { Use of immobilization } \\
(\mathrm{Y} / \mathrm{N}) \text {, } \\
\text { Use of Analgesia }(\mathrm{Y} / \mathrm{N}) \text {. }\end{array}$ & Electronic collection \\
\hline 3 weeks & $\begin{array}{l}\text { Wong-Baker FACES Pain } \\
\text { scale, } \\
\text { Use of immobilization } \\
(Y / N) \text {, } \\
\text { Days of school absence, } \\
\text { PROMIS UE, } \\
\text { EQ5D-Y } \\
\text { Economics questionnaire. }\end{array}$ & Electronic collection \\
\hline 6 weeks & $\begin{array}{l}\text { Wong-Baker FACES Pain } \\
\text { scale, } \\
\text { Days of school absence, } \\
\text { PROMIS UE, } \\
\text { EQ5DY } \\
\text { Economics questionnaire } \\
\text { Treatment satisfaction }\end{array}$ & Electronic collection \\
\hline
\end{tabular}

groups we resolved to measure pain at three days postrandomization as the primary outcome. We presented children of the YPAG several valid pain scores used in children ('Faces Pain Scale-Revised', 'Wong-Baker scale' and 'Oucher'). Children indicated a preference for the use of the Wong-Baker scale. We then tested the use of this measure, and it's electronic collection in a small feasibility study. ${ }^{14}$ This study confirmed that early pain was of primary significance related to this injury, with pain almost resolved at three weeks.

The primary outcome measure for this study is therefore the Wong-Baker scale at three days. ${ }^{15}$ This is a validated self-reported tool. It is an ordinal assessment of pain using a series of six facial-expressions to illustrate the degree of pain intensity. A numerical rating is assigned to each face (from 0 , "no hurt" to 10, "hurts worst"). It has been validated for use among children over three-years-old, including in the paediatric emergency department ${ }^{16}$; with its use being most established from five-years-old. ${ }^{4,17}$ It has been identified to be an excellent measure of pain when estimating the effect of treatment interventions in the emergency department, and is highly correlated to the visual analogue scale $(r=0.90$ $\mathrm{p}<0.001) .{ }^{16}$ Test-retest reliability is excellent, $r=0.90, \mathrm{p}$ $<0.001 .^{18}$ The Wong-Baker scale is widely used in clinical practice, forming part of the Royal College of Emergency Medicine 'Composite tool for the assessment of pain in children' produced in 2013 as part of a best practice guideline, ${ }^{1}$ and was recently specifically highlighted for use by the NICE major trauma guidelines. ${ }^{19}$

The secondary outcome measures are:

\section{Functional Recovery - Patient Report Outcomes Measurement System (PROMIS Bank v2.0) Upper Limb Score for Children Computer Adap- tive Test (CAT)}

PROMIS is a collection of patient-reported health status tools available for children and adults that were developed to be disease nonspecific in collaboration with the US National Institute for Health. ${ }^{20,21}$ These tools can be administered to healthy children as well as to children with a variety of chronic health conditions. They are selfreported from eight-years-old, and proxy-reported below eight-years-old. PROMIS is available in full (30 questions), short-form (eight questions) or as a computer adaptive test "CAT" (average eight questions). A CAT enables the answer from one question to inform the choice of the next question; so each child completing a CAT could answer a distinct set of questions to arrive at their score. Analgesia use. It is established that the analgesia used in the management of torus fractures are simple overthe-counter medications; paracetamol and ibuprofen. Patients are typically asked to purchase these over-thecounter, or out of daytime hours may be given a short supply. Information concerning the use of analgesia will be sought at a binary level to their use in the last 24 hours. Quality of life (EQ-5D-Y). This is the child-friendly version of the EQ-5D-3L, which has been especially adapted in terms of language for children aged eight to 11 years and for adolescents aged 12 to 18 years. ${ }^{22,23} \mathrm{~A}$ proxy version is available for younger children. Its age appropriateness in terms of feasibility, reliability and validity in children and adolescents has been established. ${ }^{24}$

Days of absence from school/childcare. School absence due to injury will be recorded as well as days of purchased childcare and lost working days due to child's injury.

Complications. All complications will be recorded. Particular note will be made of hospital re-attendance related to the index injury including inadequate analgesia, refracture or worsening of the fracture.

Healthcare utilization. This will be monitored for the economic analysis. Unit cost data will be obtained from national databases such as the BNF, NHS Reference Costs, and PSSRU Costs of Health and Social Care. Where these are not available the unit cost will be estimated in consultation with the Oxford University Hospitals finance 
department. NHS costs and participants'/parents' outof-pocket expenses will be recorded via a short questionnaire which will be administered at three and six weeks post-randomization.

Throughout the internal pilot phase, completion rates of outcome measures will be carefully monitored. A review of these rates will be discussed by the trial management group on a monthly basis, with potential interventions, such as paper CRFs or reminder phone calls, discussed and implemented prior to the start of the main RCT recruitment phase.

\section{Sample size}

Wong-Baker FACES scale. The primary outcome is the six-category Wong-Baker scale at three days. ${ }^{17}$ The Wong-Baker scale has a minimally clinical important difference (MCID) of one face, which was determined in the setting of the paediatric emergency department. ${ }^{16}$ The Wong-Baker scale demonstrates a very high correlation with the VAS, with each face corresponded to approximately $17 \mathrm{~mm}$ on the VAS and a clinically important difference in pain. ${ }^{16}$ Each face equates to two points on the six-category Wong-Baker scale. This trial will demonstrate equivalence of a softbandage to rigid immobilization assessing the difference in means on the Wong-Baker scale at three days post-randomization. Assuming an equivalence margin of one point (half the MCID), 90\% power, one-sided $2.5 \%$ significance, and assuming that the standard deviation (SD) is 2.3 (based on results from a feasibility study), 278 patients (139 per arm) would be required to show equivalence.

Consideration must also be made of the fact that WongBaker scale is a categorical outcome that may behave non-linearly in some instances (i.e. the magnitude of pain within the intervals is not uniform), with non-linearity more likely in younger age-groups, tending to linearity in those over eight years old. ${ }^{25}$ We therefore have powered the trial for equivalence separately in the two subpopulations (< eight years of age and $\geq$ eight years age), which is also important for secondary outcomes where some are proxy-reported for participants less than eight years and patient-reported for eight years and above. Therefore, the trial is powered separately for equivalence in the two sub-populations (four to seven-year-olds and eight to 15-year-olds). In all, 278 participants with primary outcome data in each group will be required to show equivalence.

Allowing for $20 \%$ loss to follow-up inflates the sample size to 348 in each of the sub-populations (174 per arm). Given the primary outcome is at three days postrandomization, the loss to follow-up inflation can be readily adjusted to ensure the study recruits effectively and efficiently. Sample size calculations were performed in PASS (PASS 13 Power Analysis and Sample Size Software (2014). NCSS, Kaysville, Utah, USA).

\section{Methodology}

Children will be eligible for inclusion into the trial if:

- There is radiological evidence of a torus fracture of the distal radius whereby there is a cortical deformation within the distal third of the radius but no break in the cortex. These may be associated with an ipsilateral fracture to the ulna* (the ulna fracture may be buckle, greenstick or otherwise).

- They are aged four- to 15-years-old inclusive.

- Randomization must occur at the site able to definitively treat the injury (i.e. a centre able to take the decision regarding the definitive treatment approach, which will typically be the emergency department).

* Give the anatomy of the area, most children will have an injury to both the radius and the ulna bones but, through randomization, patients with a concomitant ulna fracture will be balanced between the intervention groups.

Children will be excluded from this trial if:

- The injury is more than 36 hours old.

- The treating clinician judges that there is a cortical disruption of the radius on radiographs (i.e. a greenstick fracture).

- They have sustained an additional fracture at the time of the index fracture (with the exception of ipsilateral ulna fractures). Any child with bilateral torus fractures will therefore be excluded.

- There is evidence that the patient and/or parent/ guardian would be unable to adhere to trial procedures or complete follow-up, such as insufficient English language comprehension, developmental delay or a developmental abnormality or no access by parents/guardians to a mobile telephone with internet access.

Consenting. Recruitment will take place in a minimum of $15 \mathrm{NHS}$ trusts who treat children with torus fractures of the distal radius in the UK. Eligible patients will be identified by the clinical team. The research associate will present the patient and parents/guardian with ageappropriate participant information sheets or online study information and verbal explanation of the trial procedures. The patient/parent/guardian will then be given the opportunity to discuss any issues related to the trial with the research associate and members of their family and friends. The parent/guardian will then be asked to sign an electronic informed consent form, and children from eight years will be invited to sign an electronic assent form. Assent should be taken where appropriate, however the absence of assent does not exclude the patient from the study if consent has been obtained from the parent/guardian. If a child indicates dissent or indicates they do not what to take part, the child will not be included in the study.

Randomization. Those patients who consent to take part in the trial will have their treatment allocated using a secure, centralised, online randomization service. 
All hospital treatment areas have access to the internet so will access the randomization service in real time i.e. there will be no delay in patient treatment.

Consented participants will be randomized to one of two intervention groups $(1: 1)$ using a computer randomization service provided by the Oxford Clinical Trials Research Unit (OCTRU). Randomization allocation will be implemented using stratification by centre and age (four to seven years, $\geq$ eight years) with randomization schedules prepared by the trial statistician and embedded in the online system.

Stratification by centre will help to ensure that any clustering effect related to the centre will be equally distributed in the trial arms. The catchment area (the local population served by the hospital) will be similar for all of the hospitals; each hospital being a children's injury unit dealing with these fractures on a daily basis. All of the recruiting hospitals, and indeed all hospitals throughout the NHS, use these techniques as part of their normal practice i.e. staff will already be equally familiar with both forms of treatment. This cannot eliminate the clinician-specific effect of an individual at any one centre. ${ }^{26}$ However, since the procedures are commonplace across the NHS, many clinicians will be involved in the management of this group of patients; likely between 20 and 50 clinicians at each centre, including consultants, trainees and specialist nurses. Therefore, we anticipate that each individual clinician will only treat a handful of those enrolled in the trial, reducing the risk of a clinician-specific effect upon the outcome in any one centre.

Stratification on the basis of age will ensure that the treatments are balanced across the age groups. This will take into account differences in the properties of the primary outcome by age, with the score tending to linearity in those around eight years onwards, but behaving non-linearly for those under eight years. ${ }^{25}$ Furthermore, there is a discontinuity within the secondary outcome instruments, i.e. self-reports for those $\geq$ eight years old, and proxy-reports for those < eight years old for secondary outcomes. The trial therefore considers children < eight years, separately to those $\geq$ eight years to ensure the maximum validity of the result generated, and to maximize the generalizability of the trial results.

Post-randomization withdrawals. Participants (or their parents/guardians) may decline to continue to take part in the trial at any time without prejudice. A decision to decline consent or withdraw will not affect the standard of care the patient receives. Participants (or their parents/ guardians) can withdraw by contacting the research team by telephone or email. Upon withdrawal of the patient, any data collected up until the time of withdrawal will be retained by the research team and included in the final analysis. Withdrawn patients or patients deemed ineligible after randomization will not be replaced.
Blinding. Participants and their parents/guardians cannot be blind to their treatment. The treating clinician will of course, not be blind to the treatment they are providing. However, the treating clinical team will take no part in the follow-up assessment of the patients. The outcome data will be collected directly from the participant and/or their parents/guardians.

\section{Trial treatments}

All of the hospitals involved in this trial are familiar with both treatment techniques. All of the participants will receive analgesia at the discretion of the treating clinician as per local guidelines. In the absence of local guidelines, clinicians should adhere to the Royal College of Emergency Medicine best practice guidelines for the management of acute pain in children. ${ }^{27}$

This trial will compare two approaches to treat torus fractures of the distal radius in children.

The offer of a soft bandage immobilization and immediate discharge. A simple bandage, such as a gauze bandage or similar, will be offered to participants. The use/discontinuation of the bandage will be at the discretion of the child and their parents/guardians. The bandage technique involves application to the wrist from the middle of the forearm to the level of the metacarpophalangeal joints. Participants will be discharged from the emergency department, after randomization, without the need for outpatient follow-up (as per NICE guidance). It will be advised that the child may return to activities as pain allows, a point of contact for any ongoing concern will be provided and no specific restrictions are in place. It will be advised that the bandage should not be worn for more than three weeks. A record will be made of the duration that the immobilization is worn.

Rigid splint immobilization. A rigid splint will be applied that is either manufactured to conform to the wrist (e.g. futura splints), or is moulded onto the wrist (i.e. backslab). The study is pragmatic and the exact type of splint will not be prescribed to treating clinicians. A record will be made of the type of splint used. Treatment advice and follow-up will be as per the usual practice of the treating centre. A record will be made of the duration that the immobilization is worn.

Rehabilitation. Physiotherapy does not typically form a part in the management of these injuries, and no specific guidelines will be offered to clinicians or patients.

\section{Adverse event management}

Serious adverse events (SAE) will be entered onto the SAE reporting form and reported to the central study team. Once notified, causality and expectedness will be confirmed by the chief investigator or trial nominated clinician. Some adverse events which are foreseeable as part of the proposed treatment will not be reported on an SAE reporting form; they will be recorded on a 
complications reporting form. These events include: recall to hospital outpatient/emergency department with a diagnosis of an alternative fracture pattern, or a worsening fracture deformity (with or without the need for differing inpatient or outpatient treatment).

All participants experiencing SAEs will be followed up as per protocol until the end of the trial. All unexpected SAEs or SUSARs that occur between date of consent and six weeks follow-up point will be reported to the sponsor and ethics committee.

The end of the trial will be defined as the collection/ receipt of the last follow-up questionnaire from the last participant.

\section{Analysis}

Statistical analysis. A separate statistical analysis plan (SAP) with full details of all statistical analyses planned for the data has been published alongside this protocol. The SAP has been reviewed and received input from the Trial Steering Committee (TSC) and Data Safety Monitoring Committee (DSMC). ${ }^{28}$

Any changes or deviations from the original SAP will be described and justified in the updated SAP, protocol, final report and/or publications, as appropriate. It is anticipated that all statistical analysis will be undertaken using Stata (StataCorp, College Station, Texas, USA) or other well-validated statistical packages.

In equivalence trials a maximum clinical difference $(\Delta T)$ is pre-specified at a level within which the two treatments can be considered not to differ in any clinically meaningful way. Therefore, the null hypothesis is that a difference greater than $\Delta \mathrm{T}$ exists in either direction, H0: $\Delta \leq-\Delta \mathrm{T}$ or $\Delta \geq \Delta \mathrm{T}$ and the trial is targeted to disprove this in favour of the alternative that no clinical difference exists, $\mathrm{HA}:-\Delta \mathrm{T}<\Delta<\Delta \mathrm{T}$.

All analyses will be carried out on the intention-totreat population (that is all patients will be analyzed in the group they were randomized to regardless of actual treatment received). Analyses will be repeated for the per protocol population (patients excluded from the perprotocol population will be pre-specified in the SAP) and only if the results from both the intention-to-treat and per protocol analyses show equivalence will equivalence be claimed. ${ }^{29,30}$

The results of the analysis of the primary outcome should be one of the following:

8. The confidence interval for the difference of the two treatments lies entirely within the equivalence range, $-\Delta T$ to $\Delta \mathrm{T}$, so that equivalence may be concluded with only a small probability of error.

As well as assessing both intention-to-treat and per protocol analyses, if equivalence is demonstrated this will also form part of an additional sensitivity analysis to assess the range of potential biases that could have resulted from loss to follow-up, protocol deviations, and withdrawal.
Standard descriptive statistics will be used to describe the demographics between the treatment groups, reporting means and standard deviations or medians and interquartile ranges as appropriate for continuous variables, and numbers and percentages for binary and categorical variables. Summaries will be presented overall and separately for the two age groups. All comparative outcomes will be presented as summary statistics and reported together with $95 \%$ confidence intervals. All tests will be carried out at a 5\% two-sided significance level.

The Wong-Baker scale at three days postrandomization is the primary outcome of the study and will be compared between treatment groups as the dependent variable in a multivariate linear regression model, including all patients, and adjusting for the stratification factors and participant sex. An unadjusted $t$-test will also be undertaken. Separate analyses of patients in the two subpopulations (< eight years and $\geq$ eight years) will be undertaken using the same methodology. Additional analyses utilizing all the time-points using multi-level modelling will also be undertaken for completeness. These analyses will be performed for the available case dataset, if there is a substantial amount of missing primary outcome data (> 10\%) then sensitivity analyses investigating missing not at random scenarios will be performed. Multi-level linear regression models will also be used to analyze continuous secondary outcomes and logistic regression models will be used to analyze binary secondary outcomes.

Economic evaluation. An economic evaluation will be conducted as part of the trial to estimate cost-effectiveness. A Health Economics Analysis Plan (HEAP), providing full details of the prospective economic analysis, has been published alongside this protocol. ${ }^{28}$ The average staff time and time to deliver the rigid immobilization and soft bandage treatment will be recorded at each centre, as well as materials, and consumables involved. Data will be collected on the health service resources used in the treatment of each participant during the period between randomization and 6 weeks post-randomisation. At three and six weeks post-randomization, parents/guardians will be asked to complete economic questionnaires profiling their child's hospital (inpatient and outpatient) and community health resource use and as well as their own out-of-pocket expenditures and costs associated with their lost productivity. Unit cost data will be obtained from national databases such as the BNF and PSSRU costs of health and social care. ${ }^{31}$

Health-related quality of life will be estimated using the EuroQol EQ-5D-Y. ${ }^{22,23}$ Responses to the EQ-5D-Y will be converted into health preference scores using established methods. ${ }^{32,33}$ EQ-5D-Y responses will be valued using the most appropriate valuation set available for the trial population at the time of analysis. If necessary the adult EQ-5D-3L will be applied, in which case we will 
undertake sensitivity analysis to make sure that trial findings are not sensitive to the valuation set chosen.

Missing data will be explored and managed using similar methods to the main statistical analysis. A withintrial evaluation will be conducted from a UK NHS and Personal Social Services perspective using the FORCE trial data. ${ }^{34}$ An incremental cost-effectiveness analysis, expressed in terms of incremental cost per qualityadjusted life year (QALY) gained will be performed using bivariate regression. A further regression analysis with an interaction term will be fitted in the model to account for age as a stratification variable. This approach to agegroups differs from the primary outcome analysis because cost-effectiveness analysis is concerned with estimation rather than equivalence hypothesis testing. Results will be presented using incremental cost-effectiveness ratios (ICERs), net benefit and value of information. Furthermore, sensitivity analyses will consider the broader issue of the generalisability of the study results, including a broader societal perspective to include out-off pocket expenses borne by participants' parents/guardians, informal care provided by family and friends, and parents/ guardians income loss.

\section{Trial oversight}

The trial will be carried out in accordance with Medical Research Council (MRC) good clinical practice and applicable UK legislation using the most recently REC approved trial protocol. ${ }^{35}$ GCP-trained personnel will conduct the trial.

The day-to-day management of the trial will be the responsibility of the clinical trial manager, based at Nuffield Department of Orthopaedics, Rheumatology and Musculoskeletal Sciences and supported by the OCTRUstaff. This will be overseen by the trial management group, who will meet monthly to assess progress.

A TSC and a DSMC will be set up. The DSMC will adopt a DAMOCLES charter which defines its terms of reference and operation in relation to oversight of the trial. They will not be asked to perform any formal interim analyses of effectiveness. They will, however, see copies of data accrued to date, or summaries of that data by treatment group and they will assess the screening algorithm against the eligibility criteria. They will also consider emerging evidence from other related trials or research and review related SAEs that have been reported.

\section{Quality control}

The study may be monitored, or audited in accordance with the current approved protocol, relevant regulations and standard operating procedures by the Host organization, Sponsor or appropriate Regulatory Authorities. A Monitoring Plan will be developed according to OCTRU standard operating procedures which involves a risk assessment. The monitoring activities are based on the outcome of the risk assessment and may involve central monitoring and site monitoring.

\section{Patient and public involvement}

Patients and children were involved from the inception of the trial, including in the development of the funding application. The study was discussed in detail with members of the NIHR Young Person's Advisory Group (NIHR YPAG) who chose the logo for study from an online design competition. An ongoing commitment has been made to continue to work with this group in the production of patient-facing materials and the study dissemination plan.

To ensure ongoing patient and public involvement, a patient/carer representative will be actively involved in general trial management. In addition, a further independent patient/carer representative will become a member of the steering committee.

\section{Ethics and dissemination}

National Research Ethic Committee approved this study on the 16 November 2018 with reference number 18/ WM/0324. The study monograph for the National Institute for Health Research Health Technology Assessment will be prepared by the trial management team upon completion of the trial. A manuscript for a high impact peer-reviewed journal will be prepared simultaneously, which will allow for the results to be disseminated across the orthopaedic and emergency medicine communities, the wider medical community, NICE and policy makers. Authorship will be determined in accordance with the ICMJE guidelines and other contributors will be acknowledged. The results of this trial will substantially inform clinical practice on the clinical and cost effectiveness of the treatment of this injury. The results of this project will be disseminated to patients via patient-specific newsletters and through local mechanisms at all participating centres. Dissemination is anticipated in Winter 2020.

\section{References}

1. Fractures (non-complex). Assesment and Management. In: (UK) NCGC, ed. online, 2016.

2. Solan MC, Rees R, Daly K. Current management of Torus fractures of the distal radius. Injury. 2002;33(6):503-505

3. Charnley J, Press CU. The closed treatment of common fractures. Cambridge: Cambridge University Press, 2003.

4. Davidson JS, Brown DJ, Barnes SN, Bruce CE. Simple treatment for torus fractures of the distal radius. J Bone Joint Surg Br. 2001;83(8):1173-1175.

5. Oakley EA, Ooi KS, Barnett PLJ. A randomized controlled trial of 2 methods of immobilizing Torus fractures of the distal forearm. Pediatr Emerg Care. 2008;24(2):65-70.

6. Plint AC, Perry JJ, Correll R, et al. A randomized, controlled trial of removable splinting versus casting for wrist buckle fractures in children. Pediatrics. 2006;117(3):691-697.

7. Symons S, Rowsell M, Bhowal B, Dias JJ. Hospital versus home management of children with buckle fractures of the distal radius. A prospective, randomised trial. J Bone Joint Surg Br. 2001;83(4):556-560.

8. Williams KG, Smith G, Luhmann SJ, et al. A randomized controlled trial of cast versus splint for distal radial buckle fracture: an evaluation of satisfaction, convenience, and preference. Pediatr Emerg Care. 2013;29(5):555-559. 
9. Hamilton TW, Hutchings $\mathbf{L}$, Alsousou J, et al. The treatment of stable paediatric forearm fractures using a cast that may be removed at home: comparison with traditional management in a randomised controlled trial. Bone Joint J. 2013;95$B(12): 1714-1720$.

10. Hill CE, Masters JPM, Perry DC. A systematic review of alternative splinting versus complete plaster casts for the management of childhood buckle fractures of the wrist. J Pediatr Orthop B. 2016;25(2):183-190.

11. Jiang N, Cao Z-H, Ma Y-F, et al. Management of pediatric forearm Torus fractures: a systematic review and meta-analysis. Pediatr Emerg Care. 2016;32(11):773-778.

12. Schulz KF, Altman DG, Moher D, et al. CONSORT 2010 statement: updated guidelines forreporting parallel group randomised trials. BMJ. 2010;340:c332.

13. Moher D, Hopewell S, Schulz KF, et al. Consort 2010 explanation and elaboration: updated guidelines for reporting parallel group randomised trials. BMJ. 2010;340:c869.

14. Widnall J, Capstick T, Wijesekera M, Messahel S, Perry DC. Pain scores in torus fractures.Bone Joint Open. 2020;1-2:3-7.

15. Wong DL, Baker CM. Pain in children: comparison of assessment scales. Pediatr Nurs. 1988;14(1):9-17.

16. Garra G, Singer AJ, Taira BR, et al. Validation of the Wong-Baker faces pain rating scale in pediatric emergency department patients. Acad Emerg Med. 2010;17(1):50-54.

17. Tomlinson D, von Baeyer CL, Stinson JN, Sung L. A systematic review of faces scales for the self-report of pain intensity in children. Pediatrics. 2010;126(5):e116 8-e1198.

18. Keck JF, Gerkensmeyer JE, Joyce BA, Schade JG. Reliability and validity of the faces and word descriptor scales to measure procedural pain. J Pediatr Nurs. 1996;11(6):368-374.

19. Major trauma. Assessment and initial management. In: Excellence NIfHaC, ed. NG3, 2016.

20. PROMIS. Patient-reported Outcomes Measurement Information System. In: (U.S.) $\mathrm{NioH}$, ed., 2010.

21. Cella D, Yount S, Rothrock N, et al. The patient-reported outcomes measurement information system (PROMIS): progress of an NIH roadmap cooperative group during its first two years. Med Care. 2007;45(5 Suppl 1):S3-S11.

22. Eidt-Koch D, Mittendorf T, Greiner W. Cross-Sectional validity of the EQ-5D-Y as a generic health outcome instrument in children and adolescents with cystic fibrosis in Germany. BMC Pediatr. 2009;9:55.

23. Wille N, Badia X, Bonsel G, et al. Development of the EQ-5D-Y: a child-friendly version of the EQ-5D. Qual Life Res. 2010;19(6):875-886

24. Ravens-Sieberer U, Erhart M, Rajmil L, et al. Reliability, construct and criterion validity of the KIDSCREEN-10 score: a short measure for children and adolescents' well-being and health-related quality of life. Oual Life Res. 2010;19(10):1487-1500.

25. Oliveira A, Batalha $\mathbf{L}$, Fernandes A, et al. A functional analysis of the WongBaker faces pain rating scale: linearity, discriminability and amplitude. Rev. Enf. Ref. 2014;IV Série(3):121-130.

26. Ramsay CR, Grant AM, Wallace SA, et al. Statistical assessment of the learning curves of health technologies. Health Technol Assess. 2001;5(12):1-79.

27. Management of pain in children. Medicine TRCoE, ed. London, 2017.

28. Knight R, Dritsaki M, Mason J, Perry D, Dutton SJ. The Forearm Fracture Recovery in Children Evaluation (FORCE) trial: statistical and health economic analysis plan for an equivalence randomized controlled trial comparing soft bandage and immediate discharge versus current treatment with rigid immobilization for torus fractures of the distal radius in chiildren.Bone Joint Open. 2020;1-6:In press.

29. Chow SC, Wang H. On sample size calculation in bioequivalence trials. $J$ Pharmacokinet Pharmacodyn. 2001;28(2):155-169.

30. Christensen E. Methodology of superiority vs. equivalence trials and non-inferiority trials. J Hepatol. 2007;46(5):947-954.

31. Curtis BL. Social networking and online recruiting for HIV research: ethical challenges. J Empir Res Hum Res Ethics. 2014;9(1):58-70.
32. Brazier J, Roberts J, Deverill M. The estimation of a preference-based measure of health from the SF-36. J Health Econ. 2002;21(2):271-292.

33. Dolan P, Roberts J. Modelling valuations for Eq- $5 d$ health states: an alternative model using differences in valuations. Med Care. 2002;40(5):442--6..

34. Curtis L, Burns A. Unit Costs of Health and Social Care 2018. Canterbury. 2018. https://www.pssru.ac.uk/project-pages/unit-costs/unit-costs-2018/ (date last accessed last accessed 19 May 2020).

35. The guidelines manual. In: excellence NIfhac, ed. London, 2012.

\section{Author information:}

I. Achten, PhD, Research Manager

M. L. Costa, PhD, Professor of Trauma and Orthopaedic Surgery

D. Appelbe, PhD, Information Technology Specialist

Oxford Trauma, Nuffield Department of Orthopaedics, Rheumatology and Musculoskeletal Sciences, University of Oxford, Oxford, UK.

R. Knight, PhD Medical Statistician

S. J. Dutton, MSC, Senior Medical Statistician

Oxford Clinical Trials Research Unit, Centre for Statistics in Medicine, Nuffield Department of Orthopaedics, Rheumatology and Musculoskeletal Sciences, University of Oxford, Oxford, UK.

- J. Mason, PhD, Professor of Health Economics, Centre for Health Economics at Warwick, Warwick Clinical Trials Unit, Warwick University, Coventry, UK.

M. Dritsaki, PhD, Health Economist, Oxford Clinical Trials Research Unit, Nuffield

Department of Orthopaedics, Rheumatology \& Musculoskeletal Sciences,

University of Oxford, Oxford, UK.

- S. Messahel, MBChB, Consultant in Paediatric Emergency Medicine

J. Widnall, MBCHb, Specialty Trainee in Trauma and Orthopaedic Surgery

Institute in the Park, Alder Hey Children's Hospital, Liverpool, UK.

D. Roland, PhD, Associate Professor in Paediatric Emergency Medicine, Paediatric Emergency Medicine Leicester Academic (PEMLA) Group, Children's Emergency Department, Leicester Royal Infirmary, Leicester, UK; SAPPHIRE Group, Health

Sciences, University of Leicester, Leicester, UK.

D. C. Perry, PhD, Associate Professor of Trauma and Orthopaedic Surgery, Oxford Trauma, Nuffield Department of Orthopaedics, Rheumatology and Musculoskeletal Sciences, University of Oxford, Oxford, UK; Institute in the Park, Alder Hey Children's Hospital, Liverpool, UK.

Author contributions:

- J. Achten: Research methodology and management sections; First draft of manuscrip - R. Knight: Reviewed sample size, Carried out statistical analysis.

- S. Dutton: Reviewed sample size, Carried outstatistical analysis.

- M. Costa: Developed research question, Developed research methodology and management sections

J. Mason: Health economic evaluation.

M. Dritsaki: Health economic evaluation.

D. Appelbe: Created data management section.

S. Messahel: Created background section, Developed the research question.

D. Roland: Developed background section, developed the research question.

D. Roland: Developed background section, developed the research question.

D. C. Perry: Developed background section, Developed the research question, research methodology, and management sections.

Funding statement:

DP/MC/JA/SD/ DR/SM/JW/JM/MD obtained grant funding for this project. This project was funded by the UK NIHR HTA Programme (project number 17/23/02) and was supported by NIHR Oxford Biomedical Research Centre. Daniel C Perry is funded by a National Institute for Health Research Clinician Scientist Award (CS-2014-14-012). The funder has not been involved in the design of the study. The views expressed The funder has not been involved in the design of the study. The views expressed
are those of the authors and not necessarily those of the NHS, the NIHR, or the Department of Health.

Competing interest:

- MC is an NIHR Senior Investigator and MC and DP are members of the UK National Institute for Health Research (NIHR) Health Technology Assessment (HTA) Funding Board.

Ethical review statement:

- The National Research Ethics Committee approval was obtained on 16 November 2018 (18/WM/0324). The NIHR Health Technology Assessment monograph and a manuscript to a peer-reviewed journal will be submitted upon completion of the trial. The results of this trial will substantially inform clinical practice on the clinical and cost-effectiveness of the treatment of this injury. ISRCTN13955395.

(c) 2020 Author(s) et al. This is an open-access article distributed under the terms of the Creative Commons Attributions licence (CC-BYNC-ND), which permits unrestricted use, distribution, and reproduction in any medium, but not for commercial gain, pro- 